\title{
Religionspsychologische Biographienforschung.
}

\section{Von}

\author{
Rektor J. S c h l ü ter in Gold berg (Mecklenburg).
}

Die Religionspsychologie ist eine empirische Wissenschaft. Ihre wichtigste Aufgabe ist daher die Herbeischaffung eines möglichst vielseitigen, systematisch geordneten religionspsychologischen Materials. Erst wenn dieses vorhanden ist, kann man an die Aufstellung allgemeiner Gesetze denken. In derselben Weise, wie man in der Naturwissenschaft von der Beobachtung der Tatsachen zur Feststellung von Gesetzen fortschreitet, gilt es auch in der Religionspsychologie zu verfahren. Bevor wir über ein einigermaßen ausreichendes Material verfügen, woran es in der Gegenwart noch fehlt, hat alles Theoretisieren über Wert, Bedeutung, Stellung usw. der Religionspsychologie innerhalb der Theologie und Religionswissenschaft nur einen geringen Wert.

Die Religion tritt nun empirisch in zwiefacher Weise in Erscheinung, einmal als ein völkerpsychologisches, sodann als ein individualpsychologisches Phänomen. Einmal ist sie eine Aeußerung der allgemeinen sich entwickelnden menschlichen Seele, sodann eine Aeußerung der individuell bestimmten menschlichen Seele. Als allgemeines Phänomen kann die Religion empirisch nur auf dem Wege der völkerpsychologischen Methode erforscht werden. Diese Methode ist bisher am fruchtbringendsten von $W$. W undt angewandt worden. Er hat nicht nur ein umfangreiches religionspsychologisches Material zusammengetragen, sondern es ist ihm auch gelungen, bleibend wertvolle Gesetze und Erkenntnisse für die Erforschung des Wesens und Werdens der Religion aufzufinden. Aber dennoch ist es sicher, daB diese Methode allein nicht ausreicht, um allen religiösen Erscheinungen gerecht zu werden. Denn die Religion ist nicht nur ein völker-, sondern auch ein individualpsychologisches Phänomen, und zwar wird sie dies in steigendem $\mathrm{MaBe}$, je weiter sich die menschliche Psyche 
entwickelt, und je höher die Stufe ist, auf der die Religion steht. Auf der primitiven Religionsstufe wird man noch ganz von der individuellen Ausprägung der Religion absehen können. Hier kann sie noch angesehen werden als eine Funktion und ein Erlebnis der Gesamtseele. Von dem Augenblick an aber, wo die Religion sich nicht mehr im Kultus erschöpft, sondern wo die menschliche Seele sich so weit entwickelt hat, daß sie nach einer Subjektivierung und individuellen Erfassung der Religion verlangt, versagt die alleinige Anwendung der völkerpsychologischen Methode. Ihre Betrachtungsweise ist $\mathrm{zu}$ allgemein und zu grob, um den individuellen Gestaltungen der Religion in den einzelnen Seelen nachgehen zu können. Dies zeigt sich auch in der Völkerpsychologie Wundts. So vorzüglich ihm die Erfassung der primitiven Religion gelungen ist, so sehr versagt sein Werk, sobald es sich um die höheren Religionen handelt.

Es ist daher neben der völkerpsychologischen Methode, deren Berechtigung unbestritten bleiben soll, die Anwendung der individualpsychologischen zu fordern. Beide sollen sich nicht bekämpfen, sondern ergänzen, da beide imstande sind, Wertvolles für die Erkenntnis des religiösen Phänomens beizutragen. Mit Recht bemerkt $\mathrm{K}$ a l w e it (Rel. in Gesch. u. Gegenw. IV, 22Io): "man soll bei dem gegenwärtigen Stand der Arbeit nicht engherzig sein und nur für eine einzige Auffassung ausschließliches Recht in Anspruch nehmen. Weil wir noch ganz und gar in den Anfängen stehen, ist jede Herbeischaffung religionspsychologischen Materials dankbar zu begrüßen."

Es ist freilich zuzugeben, daB die Fehlerquellen bei der individualpsychologischen Methode größere sind als bei der sozialpsychologischen. Die letztere schöpft ihr Tatsachenmaterial aus der objektiven Erscheinungswelt der Religion, die sich sicher feststellen läßt, und etwaige Fehler können erst bei der Deutung und Verwendung der Tatsachen entstehen. Die erstere dagegen entnimmt ihre Tatsachen den subjektiven Erlebnissen der einzelnen religiösen Individuen. Da diese aber bei einem jeden verschieden auftreten, fehlt jede Möglichkeit, ihre Richtigkeit nachzuprüfen. Dazu kommt, daß es sehr schwer ist, das eigene religiöse Erleben adäquat wiederzugeben. Sehr vielen, die die nötige sprachliche und psychologische Schulung besitzen, mangelt es an dem nötigen eigenen religiösen Erleben, und sehr vielen, die dies besitzen, fehlt wieder die nötige sprachliche und psychologische Schulung.

Aber dennoch können die hier angedeuteten Schwierigkeiten den Wert der individualpsychologischen Methode nicht aufheben. Es ist möglich, durch sorgfältige Prüfung und Sichtung des Quellen- 
materials die Fehlerquellen auf ein Mindestmaß einzuschränken und so wertvolle religionspsychologische Resultate $z$ erzielen.

Die individualpsychologische Methode kann wieder ein zweifaches Verfahren einschlagen. Man wendet sich entweder an möglichst viele gegenwärtig lebende Individuen und erbittet von ihnen Antworten auf einen ihnen zugestellten Fragebogen, oder man richtet die Untersuchung in erster Linie auf die Klassiker der Religion in Gegenwart und Vergangenheit. Das erste Verfahren ist in Amerika von $\mathrm{Leuba}$, $\mathrm{St}$ a r b u c k u. a., in Deutschland von $\mathrm{P} f$ en $\mathrm{n}$ i g s d or f eingeschlagen worden. Es ist nicht zu leugnen, daß es imstande ist, wertvolles religivuspsycinuiugiscines Iríaichia' zu liefen. Wrenn das Taisachennaterial umfassend und vorsichtig genug aufgestellt ist, so vermag man auf diesem Wege ein annähernd adäquates Spiegelbild der subjektiven Religion in der Gegenwart zu gewinnen. Aber man darf doch die Grenzen dieser Methode nicht verkennen. Es kann keinem Zweifel unterliegen, daß bei dieser Methode die Fehlerquellen besonders groß sind. Der Forscher kennt die meisten seiner Studienobjekte nicht und hat gar keine Möglichkeit, nachzuprüfen, wieviel Fehler bei der Beantwortung der von ihm gestellten Fragen absichtlich oder unabsichtlich gemacht werden. Außerdem laufen die Antworten von einem sehr willkürlich zusammengewürfelten Kreis von Menschen ein, dessen äußere und innere Verhältnisse dem Forscher unbekannt sind. Endlich muß diese Methode sich in erster Linie an Durchschnittsmenschen wenden, die ja zwar die Mehrzahl der religiösen Individuen ausmachen, in denen aber doch die Eigenart der Religion in ihrer Kraft und Stärke, ihrem Kampf und Sieg nicht in so charakteristischer Weise hervortritt wie bei den Klassikern der Religion.

Deren religionspsychologische Erforschung muß daher die zweite Form der individualpsychologischen Methode wählen. In dieser Beziehung ist bisher am meisten von J a m e s geleistet. Er will an besonders charakteristischen Einzelbeispielen des religiösen Lebens die Eigenart und die Grundtypen desselben aufzeigen. An diese Methode »der ausgezeichneten Fälle» schließt sich die Arbeit der religionspsychologischen Arbeitsgemeinschaft ${ }^{1}$ ) in vieler Hinsicht an. Auch sie will die Religion in ihrer Mannigfaltigkeit an möglichst vielen »ausgezeichneten Fällen "studieren und so die Religion in ihrer Eigenart erfassen, ohne sie von vornherein allgemein-psychologischen oder physiologi-

I) Vgl. Zeitschrift für Religionspsychologie Bd. IV (IgII) S. 2 I9 ff.: »Bericht über die Begründung einer religionspsychologischen Arbeitsgemeinschaft»; ferner Bd. V (1912) S. 394 ff.: "Die Verwendung von Fragebogen in der Religions. psychologie*. (Anm. d. Herausg.) 
schen Gesetzen unterzuordnen. Aber dennoch unterscheidet sie sich in wichtigen Punkten von James. Sie lehnt seine pragmatistische Philosophie $a b$ und vermeidet alle verfrühten allgemeinen Wertund Wesensurteile über die Religion. Sie erstrebt eine allseitigere und systematischere Benutzung der Quellen. Während James offenbar die exzentrischen Erscheinungen bevorzugt und seine religionsphilosophische Anschauung oft die Auswahl bestimmt, will die religionspsychologische Biographienforschung möglichst vollzählig das vorhandene Material zusammentragen und sichten, unbekümmert um religionsphilosophische Theorien und Konsequenzen. Sodann will sic die religiösen Persönlichkeiten im Zusammenhang ihres natürlichen und religiösen Milieus verstehen und nicht wie James die Einzelreligiosität isolieren. Endlich will sie die ganze religiöse Entwicklung ihres Objekts ins Auge fassen und die Untersuchung nicht wie James auf einzelne Seiten des religiösen Lebens beschränken. Damit dies möglich ist und zugleich eine gewisse einheitliche Bearbeitung garantiert wird, ist ein Fragebogen aufgestellt, der auf eine normale religiöse Entwicklung Bezug nimmt. Bei der mannigfachen Verschiedenheit der Entrvicklung der religiösen Individuen ist er nicht als ein bindendes Schema, sondern als allgemeines Orientierungsmitiel für die Mitarbeiter gedacht.

Man hat die Berechtigung dieser Methode bestritten aus Gründen religiöser Pietät und Keuschheit. Es verletze das religiöse Gefühl, die subjektiven Vorgänge der religiösen Seele in das Licht der Oeffentlichkeit, der wissenschaftlichen Beobachtung und Statistik zu ziehen. Diesem Vorwurf ist entgegenzuhalten, daß die meisten der zu behandelnden Persönlichkeiten selbst schon in Aussprüchen, Schriften und Briefen ihr Innenleben der Oeffentlichkeit preisgegeben haben, und daß niemandem Geheimnisse entlockt werden sollen, die er für sich behalten will. Sodann aber ist gar nicht einzusehen, inwiefern durch wissenschaftliche Bearbeitung die religiösen Lebensäußerungen sollten profaniert werden. Mit demselben Recht könnte man sagen, es sei unwürdig, exakte exegetische und textkritische Untersuchungen über die hl. Schrift anzustellen, oder den Inhalt der christlichen Erfahrung in die Kategorien und Begriffe der Dogmatik $z u$ bringen.

Wenn endlich der $W$ e $r t$ dieser Methode in Zweifel gezogen wird, so muß man von vornherein zugeben, da $B$ man allerdings auf diesem Wege nicht das ganze Phänomen der Religion von allen Seiten erfassen oder erklären, geschweige denn das Wesen der Religion auffinden oder die Frage nach ihrer Wahrheit lösen kann. Alles dies 
greift über den Rahmen dieser Methode, aber zugleich über den Rahmen jeder empirischen Forschung hinaus. Aber was eine empirische Wissenschaft überhaupt leisten kann, dafür ist eine solche Biographienforschung hervorragend geeignet. Es ist ihre erste Aufgabe, möglichst viele und möglichst vielseitige Tatsachen des religiös-psychischen Lebens aufzusuchen und bereitzustellen, ihre zweite Aufgabe, in diesen Tatsachen gewisse RegelmäBigkeiten und Gesetze aufzuzeigen und nachzuweisen. Um nur einige Beispiele zu nennen: ein umfangreiches Tatsachenmaterial wird GesetzmäBigkeiten oder verschiedene Typen der religiösen Entwicklung, gewisse Korrelationen

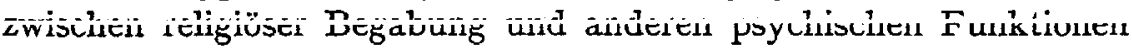
erkennen lassen. Aber mit der Sammlung individueller Erlebnisse, der Aufzeigung bestimmter Typen und der Auffindung gewisser GesetzmäBigkeiten ist die Aufgabe der religionspsychologischen Biographienforschung erschöpft. Der Religionsmetaphysik einerseits, der praktischen Theologie andererseits muß es überlassen bleiben, die Ergebnisse einer solchen empirischen Forschung für ihre Zwecke und mit ihren Methoden zu beleuchten und zu verarbeiten.

Aber eben zu der beschriebenen Biographienforschung ist eine Arbeitsgemeinschaft vieler unentbehrliche Voraussetzung. Daher ist es der wesentliche $Z$ weck dieser Seiten, zur Mitarbeit an diesem $Z$ weig der Religionspsychologie aufzufordern. Für eine solche Mitarbeit sind folgende Grundsätze maßgebend.

Grundsätze einer religionspsychologischen Biographienforschung ${ }^{1}$.

$\mathrm{Ziel}$ der Arbeit. Die religionspsychologische Biographienforschung will die religiöse Entwicklung möglichst vieler selbständiger Persönlichkeiten feststellen und sie nach einheitlichen Gesichtspunkten und Methoden zur Darstellung bringen. Von dieser Arbeit erhofft sie nicht nur interessante und wertvolle Einblicke in das religiöse Leben vieler Individualitäten, sondern auch die Gewinnung allgemeiner Erkenntnisse und Gesetze für den Verlauf der religiösen Entwicklung überhaupt.

Quellen der Bearbeitung:

I. Ueberall, wo es irgend angängig ist, sind eigene Aufzeichnungen der betreffenden Persönlichkeit, wie Autobiographien, Briefe, Tagebücher usw. zugrunde zu legen.

I) Abdruck dieser "Grundsätze mit Quellenangabe ist sehr erwãnscht. - Sonderabzüge sendet auf Wunsch Rektor J. Schlüter, Goldberg (Mecklenburg), an den auch alle Zuschriften und Anfragen in dieser Sache zu richten sind. 
2. In zweiter Linie ist die Literatur über die betreffende Persönlichkeit $\mathrm{zu}$ benutzen. Es kommen in Betracht Aeußerungen von Zeitgenossen und Nachlebenden, Biographien, spezielle Abhandlungen über die Religion des Behandelten usw.

Met hode der Bearbeit ung. Jeder Bearbeiter durchforscht zunächst ohne Rücksichtnahme auf den Fragebogen die von ihm übernommene Biographie und versucht, sich einen allgemeinen Ueberblick und ein allgemeines Urteil über die religiöse Entwicklung der betreffenden Persönlichkeit $\mathrm{zu}$ verschaffen. Ist ihm dies gelungen, so versucht er an der Hand des ihm zugestellten Fragebogens, jedoch ohne ihn als bindendes Schema zu benutzen, möglichst adäquat die religiöse Entwicklung in ihren einzelnen Formen und Stufen darzustellen. Soweit es möglich ist, schließt er sich dabei an den Gang des Fragebogens an. Fragen, die in dem betreffenden Leben keine Rolle spielen, oder über die keinerlei Aufschluß zu erhalten ist, werden überschlagen. Wichtige Tatsachen, die in das Fragebogenschema nicht passen, werden besonders aufgeführt. Auch ist überall, soweit es von Bedeutung ist, Bezug zu nehmen auf die allgemeinen psychischen und sonstigen Vorbedingungen des religiösen Lebens. (Lebensalter, Lebenslage, Beschäftigung, Gesundheit, Bildung, Verkehr, Temperament usw.)

Alle Antworten sind durch genaue Zitate mit Angabe ihres Fundortes zu belegen. Mutmaßungen und Schlüsse sind als solche zu kennzeichnen, besonders wichtige Aussprüche sind wörtlich anzuführen. Endlich ist zu beachten, da $B$ die einzelne Bearbeitung keine vollständige und ausgeführte religiöse Entwicklungsgeschichte bieten soll, sondern nur eine Darstellung der religionspsychologisch wichtigsten Hauptmomente derselben. Eine allzu große Ausdehnung der Arbeit ist daher zu vermeiden.

Die fertige Ausarbeitung besteht

I. aus einer ganz kurzen, sich auf die äußeren Daten beschränkenden Biographie,

2. aus einer Angabe der benutzten Literatur,

a) der oben sub $I$,

b) der sub 2 genannten,

3. aus einer ausführlichen Beantwortung des Fragebogens.

$$
\text { Frage bogen. }
$$

A. Kind he it.

I. In welcher Umgebung wächst das Kind auf?

a) Welches ist das natürliche, b) das religiöse Milieu?

2. Welchen EinfluB hat die Erziehung auf die religiöse Entwicklung des Kindes?

3. Wann und in welcher Form traten zuerst religiöse Erlebnisse und Vorstellungen in der Seele des Kindes auf?

a) Von welcher Person oder welchem Ereignis gehen sie aus?

b) Welcher Art sind diese Eindrücke? 
c) Haben sie dauernde Bedeutung für das Gefühls-, Willens- oder Vorstellungsleben?

4. Entwickeln sich in dem Kinde besondere Eigenschafien, Neigungen usw., die von Bedeutung für die religiöse Entwicklung sind?

5. An welche einzelnen Erlebnisse knüpfen sich im Vrrlauf der kindlichen Entwicklung Eindrücke religiöser Art und von welcher Art und Bedeutung sind sie?

6. Treten schon in der Kindheit $Z$ weifel auf, und woran schließen sie sich an?

7. Gegen welche Fehler kämpft das Kind, und welche Rolle spielen dabei religiöse Vorstellungen?

8. Welcher Art ist das kindliche Gebetsleben, und welche Erfahrungen werden dabei gemacht?

9. Wann und wie tritt die Person Jesu für das religiöse Leben des Kindes in Erscheinung?

Io. Welche Wirkung hat der Religionsunterricht?

II. Beteiligt sich das Kind an der kirchlichen Sitte? Warum? Gern oder ungern? In welcher Weise ist es innerlich dabei beteiligt?

I2. Kennt das Kind eine religiöse häusliche Sitte? Ist es innerlich an ihr beteiligt? Welchen Einfluß hat sie auf die religiöse Entwicklung des Kindes?

13. Welchen Eindruck macht und hinterläßt die Konfirmation?

I4. Weitere Mitteilungen.

B. Uebergangszeit.

I. Wie gestaltet sich im allgemeinen in dieser Zeit das religiöse Leben (Gebet, Sünde, Kirche, religiöse Lektüre, Umgang mit Menschen usw.)?

2. Welche Faktoren wirken neu auf die religiöse Entwicklung ein?

3. Treten $Z$ weifel oder Anfechtungen auf ?

a) Wann?

b) Wovon gehen sie aus (Person, Ereignis, Buch usw.)?

c) Welcher Art sind sie?

d) Wirken sie drückend oder befreiend auf das seelische Leben?

e) Wurden sie beseitigt? Wodurch? Warum nicht?

4. Welche Bedeutung hat das sexuelle Leben für die religiöse Entwicklung?

5. Ist die religiöse Entwicklung eine gradlinig fortschreitende oder führt sie durch deutliche Krisen und Erschütterungen hindurch ?

a) Wodurch werden sie hervorgerufen?

b) Welche äußeren und inneren Zustände gehen ihnen voraus?

c) Welchen Verlauf nehmen sie?

d) Welche Vorstellungen werden aufgegeben, welche zurückgestellt, und welche treten jetzt in den Vordergrund?

6. Weitere Mitteilungen. 


\section{Z e it der Reife.}

I. Welches ist das allgemeine Ziel (Religion, Konfession, Sondergemeinschaft, Weltanschauung usw.), zu dem die religiöse Entwicklung in der Zeit der Reife geführt hat?

2. Werden auch in dieser Zeit noch wichtige Krisen durchgemacht? In welchem Alter treten sie auf, und wie verlaufen sie?

3. Wie gestaltet sich im einzelnen die Religion des reifen Mannes?

a) Wie steht er zu den Lehren der offiziellen Kirche?

b) Welche Bedeutung hat für sein religiöses Leben die Kirche mit ihren Einrichtungen und Dienern? Was zieht ihn an, was stößt ihn ab?

c) Welche Bedeutung hat die religiöse Literatur (Bibel, Gesangbuch, Katechismus, religiöse Romane usw.)?

d) Sind besondere Persönlichkeiten der Gegenwart oder Vergangenheit die ausgesprochenen Führer?

e) Welche Bedeutung hat die Person Jesu?

f) Welche Bedeutung haben Kunst, Natur, Wissenschaft usw.?

g) Welche Bedeutung haben die Wechselfälle des Lebens?

h) Welche Bedeutung hat das öffentliche und berufliche Leben?

i) Welcher Art ist das Gebetsleben? Frei oder gebunden? Regelmäßig oder nur bei besonderen Anlässen? Welches ist der erfahrene innere und äußere Erfolg?

k) Treten Erlebnisse irgendwie mystischer Art auf, und wie verlaufen sie im einzelnen?

1) Inwiefern beeinflußt die Religion das sittliche Leben?

m) Wie wird Sünde, Schuld und Gnade erlebt?

n) Wie äußert sich die Religion angesichts und während des Sterbens?

4. Weitere Mitteilungen.

5. Welches sind die Hauptmerkmale, die den Typus der Frömmigkeit der behandelten Persönlichkeit charakterisieren?

Folgende Biographienliste ist bisher aufgestellt; Ergänzungen sind willkommen; Biographien, für die schon ein Bearbeiter gewonnen ist, sind mit * bezeichnet.

\begin{tabular}{lll} 
Alacoque & Boos, M. & Darwin * \\
Alline, Henry & Bourignon & Daudet \\
Amiel & Bräker, Uli * & Dickens \\
Andersen * & Brater, Pauline * & Dietrich, Amalie \\
Arndt, E. M.* & Bülow, Gabr. v. & Dörpfeld \\
Augustin * & Bunyan & Drummond, H. \\
Bach, J. S. & Calvin & Ebner-Eschenbach,M.v.* \\
Beyschlag & Carlyle & Eckehart \\
Bismarck * & Carmen, Sylva & Egidy, M. v. \\
Blumhardt & Coleridge & Eliot, George \\
Boehme, J. * & Cromwell * & Ellis, William \\
\multicolumn{1}{|l}{ Archiv für Religionspsychologie I. } &
\end{tabular}




\begin{tabular}{|c|c|c|}
\hline Emerson * & Kierkegaard * & Roon, v. \\
\hline Emmerich, A. K. & Kingsley & Rosegger \\
\hline Feuerbach, L. * & Kügelgen * & Rousseau \\
\hline Fichte * & Lagarde * & Savonarola * \\
\hline Fliedner & Lavater * & Schäffle \\
\hline Fontane & Liliencron, D. v. & Schleiermacher * \\
\hline Fox, George * & Lincoln & Schönaich-Carolath \\
\hline Franke, A. H.* & Livingstone & Schubert, G. H. v. * \\
\hline Franklin, Benjamin & Löhe * & Schurz \\
\hline Friedrich d. Große & Longfellow & Semler \\
\hline Franz von Assisi & Loyola, I. v. * & Sieveking, Amalie \\
\hline 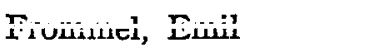 & LüLtha-ardt, $A$. & Silesius, A. \\
\hline Garfield & Luther & Stilling \\
\hline Gifford & Maeterlinck * & Stöckei \\
\hline Goethe & Malvida v. Meysenburg & StrauB, D. Fr. \\
\hline Gött, Emil & Markgräfin vonBayreuth & Strindberg \\
\hline Hase, $\mathrm{K}, \mathrm{v}$. & Meyer, C. F. * & Suso \\
\hline Hauser, Kaspar * & Molinos & Tennyson \\
\hline Hamann * & Moltke & Tersteegen \\
\hline Hamilton & Monod & Therese, Hl. \\
\hline Harless & Moritz * & Thoreau \\
\hline Hauptmann, G. & Mörike & Tolstoi * \\
\hline Hebbel & Musset & Trine \\
\hline Helene von Orleans & Napoleon * & Wagner, R. \\
\hline Herder & Nathusius & Washington \\
\hline Heyse, P. & Nietzsche ${ }^{*}$ & Watson \\
\hline Hilty * & Oberlin & Wesley \\
\hline Hoensbroech & Pascal & Whitefield \\
\hline Hülshoff, A. v. Droste ${ }^{*}$ & Paulsen & Whitmann, W. * \\
\hline Humboldt, W. v. * & Peabody & Wichern * \\
\hline Huntington & Pestalozzi & Wilhelm I. \\
\hline Irving & Perthes, Carol. & Zinzendorf * \\
\hline Jefferies & Perthes, Friedr. & Zwingli \\
\hline Keller, Helen & Richter, L. * & \\
\hline
\end{tabular}

Biographische bzw. autobiographische Romane:

Carlyle, Sartor Resartus * De Wette, Weihe des Zweiflers * Ernst, O., Asmus Semper Goethe, Bekenntnisse einer schönen Seele *
Frenßen, Jörn Uhl; - Klaus H. Baas Hauptmann, G., Der Narr in Christo Kabisch, Gottes Heimkehr *

Keller, G., Der grüne Heinrich Krüger, H. A., Gottfried Kämpfer. * 